\title{
Beyond Task-technology Fit: Exploring Network Value of Blockchain Technology Based on Two Supply Chain Cases
}

\author{
Günter Prockl ${ }^{\mathrm{a}}$, Dominik Roeck ${ }^{\mathrm{b}}$, Thomas Jensen ${ }^{\mathrm{a}}$, Somnath Mazumdar ${ }^{\mathrm{a}}$, Raghava Rao Mukkamala ${ }^{\mathrm{a}, \mathrm{c}}$, \\ ${ }^{\mathrm{a} C}$ Copenhagen Business School, Denmark, ${ }^{\mathrm{b}}$ University of St.Gallen, Switzerland, ${ }^{\mathrm{c}}$ Kristiania University College, Norway
}

\begin{abstract}
Despite the popularity of blockchain technology in the supply chain domain, cases with adoption beyond the pilot phase are limited. Even though technology fit is essential for blockchain adoption, we find network fit to be equally important for participating companies in a network. This research explores how the network affects value creation beyond a technology fit. Studying two cases, one from the gemstone industry and another from the shipping industry, we use the task technology fit model, network effects, and structural embeddedness as theoretical lenses to explore the fit that leads to the success of blockchain adoption. Our investigation reveals the task technology fit as a prerequisite and shows central organizations acting as initiators in the early phase, trying to extend the network in subsequent phases. Our investigation indicates that the network fit, autonomy, and equivalence of the organizations contributed to the successful adoption of blockchains.
\end{abstract}

\{gp,tj,sma,rrm\}.digi@cbs.dk, dominik.roeck@unisg.ch

\section{Introduction}

The disruptive nature of Blockchain technology came into the limelight when cryptocurrencies (such as Bitcoin) were introduced as decentralized peer-to-peer digital cash systems which can run without the intervention of any central actors like banks and other financial institutions. After the cryptocurrencies, the supply chain domain is one of the early adopters of the blockchain due to the premise that blockchain characteristics such as immutability, transparency, and trust can help to solve some of the challenges faced by the supply chain domain. Blockchain has the potential to change how stakeholders share supply chain information via a more trusted, secured, and accessible over a distributed ledger [12]. Some of the supply chain processes where the transparency and traceability provided by the blockchain technology can bring transformation are: improving product safety and security, fight against counterfeit products, enhancing inventory management and replenishment, decreasing transaction costs, reducing the role of intermediaries, and many others [3].

Even though the technological features of blockchain technology are suitably fitted for harnessing the benefits in the supply chain domain, the number of successful supply chain projects that adopted blockchain and went beyond the pilot study are quite limited [19]. Sternberg et al. [19] argued that the success of blockchain-based applications in the supply chain dependent on the number of actors or the critical mass of the companies that are collaborating in the network. Furthermore, the authors have also identified that in the case of an inter-organizational setting, there exist several tensions between the positive and negative driving factors for blockchain adoption, which need to be resolved for successful adoption. However, it is interesting to explore where does the actual value creation comes from in the case of blockchain adoption in an inter-organizational setting. Does it come from the technology fit, i.e., matching the system's requirements with the technological features? Or else does it come from network participants, i.e., how well the participating actors collaborate to co-create the value?

In this research, we explore these aspects with the help of two successful case studies from the supply chain domain, where the blockchain solutions have been gone beyond the pilot studies and adopted in the production setting. The first case study is in the gemstone industry, and the solution is built on the Provenance Proof Blockchain that leverages blockchain technology to provide transparency, authenticity, and integrity along the supply chain from the remote mines to the consumer. The second case study is in the shipping industry, where the TradeLens platform is built on Hyperledger Fabric blockchain technology. It provides transparency by sharing real-time shipping information for the containers' journey across multiple organizations in the supply chain, e.g., from an origin in China to a destination in Europe. We propose the following research question to understand the interplay 
between the technology fit and network effects among these case studies.

In the case of blockchain technology adoption, what does the fit mean when we investigate from the network point of view?

To address the research question, we apply Task Technology Fit, Network Effects, and Structural Embeddedness as theoretical lens to investigate to what extent the adoption of blockchain technology created value to respective stakeholders. Investigating two blockchain case studies, one from the gemstone industry and another from the shipping industry, we explore how the technology fit and network effects contributed to the value appropriation. In the next section, we will present theoretical background along with a brief review of related literature, and then we present the two case studies in section 3. In section 4 we will present our analysis and discussion, followed by a conclusion in the end.

\section{Theoretical Background}

This section presents related literature, followed by an elaboration on our theoretical lens for this paper, containing three theoretical frames: Task Technology Fit, Network Effects, and Structural Embeddedness.

\subsection{Related Literature}

Several publications $[14,3]$ explore the use of blockchain technology in the logistics and supply chains domain. The research work of Herm and Janiesch [10] reveals and prioritizes requirements for a blockchain-based collaboration platform in supply chain networks (SCN). Saberi et al. [16] identify four adoption barriers: inter-organizational, intra-organizational, technical, and external barriers, which can reduce the advantages of using blockchain in supply chains. Straubert et al. [20] took an interesting view that tracking and tracing in supply chain management using private permissioned blockchain does not offer any extra advantage compared to the existing technologies. They also claim that public permissionless blockchains can provide new functionalities, but all participants must be pseudonymous and must have read/write/verify permissions. Another research work by Blossey et al. [1] identifies three primary technical benefits: transparency, automation, and validation in using blockchain to integrate the supply chains. Seebacher and Maleshkova [17] proposed a layer-based model for capturing the blockchain-supported supply chain network for modeling the use cases of traceability and anti-counterfeiting of products, based on a business model, network composition, and technical implementation. Similarly, another research work by Mazumdar et al. [13] explores the usage of a blockchain-IoT-based framework for documenting the product life cycle with IoT devices attached to physical devices. Finally, Farshidi et al. [7] developed a multicriteria decision-making problem and introduced a decision model for the blockchain platform selection problem based on the technology selection framework.

Such existing research focuses on technical properties and resulting capabilities of blockchain technology. Our paper explores blockchain adoption from an entirely different perspective. In our research work, we take a network perspective view and investigate to what extent the adoption of blockchain technology created value to various stakeholders in the network. Beyond Task Technology Fit, we apply the lenses of Network effects and Structural Embeddedness that have been used in different context to research actors positions and resulting value creation.

\subsection{Task Technology Fit}

We intend to investigate the performance of technology, here in particular blockchain technology, expressed as adoption by potential users for a specific purpose. To explain and thus predict such adoption of technology, the framework of task-technology fit (TTF) [9] is well-established and frequently used, among similar models such as Technology Acceptance Models [4], and the Unified Theory of Acceptance and Use of Technology (UTAUT) models [22]. TTF helps to predict the adoption and application of a specific technology for a specific task based on a concept of fit. With this line of reasoning, TTF shows characteristics of a variance theory [21]. It assumes a causal link between the construct fit and the appropriation and, consequently, the application of the technology. Fit as such describes either consistency in terms of covariation, or it may be framed as multidimensional gestalt expressing congruence or adherence vs. deviation to a proposed profile [23]. In its original setup, TTF is single actor-oriented and is assessing technology fit from the viewpoint of an individual user. Though this implies certain limitations, TTF has been augmented towards the assessment of technologies for groups [23], and it is increasingly discussed in the context of network technologies such as inter-organizational systems [16].

We understand the existence of such a kind of task-technology-fit as a necessary condition prior to any organization's users' adoption of blockchain technology. However, about blockchains' character of being a network technology, we do not see this necessarily as a 
sufficient condition. Different from an application for an individual user, in a network of different involved actors, there is no clear causality between fit and appropriation. Perceived fit and appropriation result from a dynamic process and mechanisms influenced by the scope and diversity of involved actors and their interpretation in their context. In a network view, fit thus requires elements of a process theory [21] which is as such, however, not included in the TTF concept.

\subsection{Network Effects and Structural Embeddedness}

Consequently, there is a need to reflect on influencing factors towards this process of evaluating benefits and sacrifices [15], which are perceived and influenced differently by the role and number of involved actors and their diverse assessment of resulting value. Such value is not static but an episodic outcome in an ongoing process, increased by network benefits and reduced by network sacrifices, i.e., by the dynamics and effects resulting from being a part of the network. Already Katz and Shapiro [11] refer to network externalities to investigate the likelihood of rivaling technologies adoption. Accordingly, in the absence of a sponsor, a superior technology of today is likely to sustain dominating. However, when there is a sponsor involved, also the inferior one of two rival technologies may gradually dominate. The more diverse the actors in the ecosystem are, the more likely is it that fit is perceived differently by them. The more central an actor is within the network, the higher is the potential influence on the outcome. Next to the diversity of actors, this emphasizes actors' centrality and governance legitimization within an institutional setup. Dong et al. [6] introduced, for instance, a concept named institutional distance as a summation of normative, cognitive, and regulative distances to investigate the joint performance of collaborative firms.

In our approach, we refer to the concept of structural embeddedness as presented by Gnyawali and Madhavan [8], describing the position of actors in regards to access to assets, information, and status of the actor. Embeddedness is deducted from the actors' role in the context of the specific industry network description and the network, i.e., here, the blockchain, under consideration. Embeddedness appears in particular interesting for our purpose as it specifies how the structural properties of cooperative networks influence the competitive dynamics in the network. This relates on a firm level to a description of behavior as a consequence of centrality and autonomy of the actors and, in particular, differences among them.
Pairwise comparison implies a view on the equivalence of roles and positions, and on a network level, embeddedness refers to a description of network density, i.e., the interconnection among the actors in the network [8]. Our assumption is that the greater the inter-connectedness, the greater the density, and the faster the flow of information and resources in the network, the more business value can be generated. Further, the more actors and organizations connected creates the network effect, which produces exponential growth of both the network and as well in the generation of business values. With higher density, behavioral patterns should develop more easily but as well the facilitation of sanctions and counteraction to egoisms. The denser it is, the more it is expected that the network functions as a closed network and is showing a less competitive environment. Increasing density thus potentially mitigates effects of centrality and autonomy and thus resembles the dynamic effects of network externalities towards self-enforcing mechanisms of network patterns of behavior. While seeing density in principle with a positive effect on value, it may be also questioned whether there is not some level of saturation or even counter effect when density and size of the network become gradually too large.

As illustrated in Figure 1, we altogether frame appropriation as a result of an evaluation process that combines three different sources of value created by a specific technology. Task value stems from task-technology-fit in its classic understanding. Relationship value refers to the cooperative and competitive positions of specific actors within the relevant network. Network value finally is different from relationship value as it is not a consequence of the position in the network but a result of being in the network and benefiting or suffering from network dynamics as expresses in network effects as a whole.

\section{Case Studies}

This section will first present the blockchain adoption case study from the colored gemstone industry, and then we will switch to the TradeLens case study from the shipping industry.

\subsection{Case Study: Colored Gemstone Industry}

Industry: The colored gemstone industry reveals three peculiarities. First, the stones vary largely in their value per carat, depending on the characteristics of the stone, such as scarcity, quality, origin, and customer attractiveness. Hence, assessing the characteristics of a stone is key for evaluating the value of a colored gemstone. Second, the value chain in the 


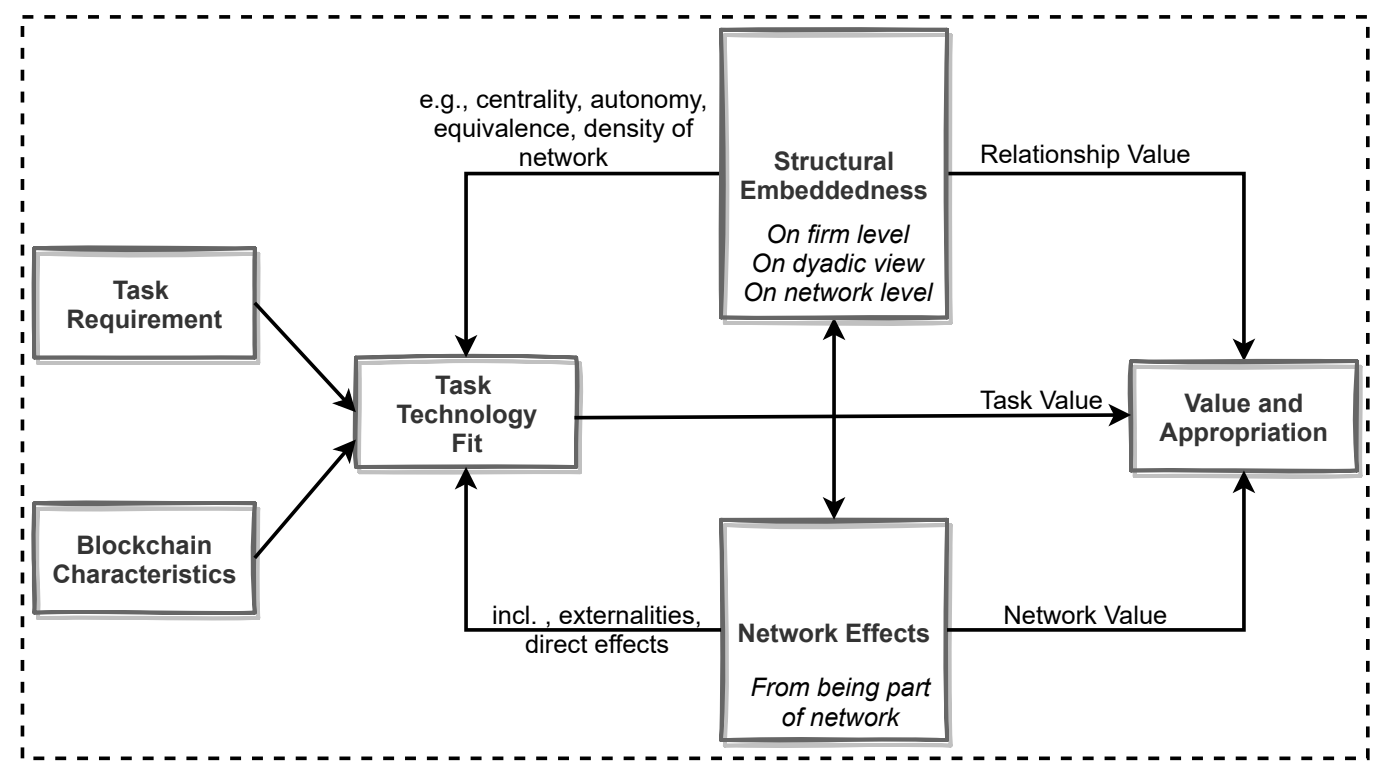

Figure 1. Conceptual framework on drivers of value and appropriation in blockchain networks

colored gemstone industry is highly complex and heterogeneous. There are many artisanal mines and only a few large-scale mines with heavy machinery on the mining stage. These are followed by traders that have either a regional, national, or international customer networks, refiners with diverse specializations in terms of cut and treatment, exporters, regional, national, and international wholesalers, jewelers, and retail brands [18]. Third, the transactions within the industry are based on mutual trust and reputation between transaction partners (e.g., miners and regional traders). A large volume of the trade is informal with limited transparency for external parties, causing substantial challenges for financing, insurance, and ethical standards. The previously described three peculiarities turned into a burden given the lack of transparency in the industry. First, for end-customers or upstream actors (e.g., retail brands), the actions of previous stages in the value chain are not transparent given the complexity and heterogeneity of the value chain as well as the informal transactions. Hence, buyers fear to finance unethical or ecological harmful actions in the downstream value chain. Second, end-customer as layperson having a hard time assessing the characteristics of a stone and its value. Consequently, end-customers are expecting more transparency on aspects such as origin, authenticity, and physical qualities.

Blockchain application: Against this backdrop, Gübelin Gem Lab ${ }^{1}$ and Everledger ${ }^{2}$ introduced

\footnotetext{
${ }^{1}$ https://www.gubelingemlab.com/en/

${ }^{2}$ https://www.everledger.io/
}

the Provenance Proof Blockchain ${ }^{3}$ for the colored gemstone industry. The blockchain solutions allow creating a digital twin for each colored gemstone and storing all relevant information (e.g., physical dimensions, color, cut) along the value chain. Each stage in the value chain has its own role and enables to upload of role-specific information. The gemstone owner can access this information and thereby trace back the stone to its origin, gain an overview of all steps within the value chain, and verify the authenticity of the gemstone. Over 500 gemstone actors from mine join the blockchain initiative to ring, accounting for a 500,000 colored gemstones that are recorded in the blockchain by July 2021 .

Technology fit: The Provenance Proof blockchain addresses the task of providing more transparency for the colored gemstone industry. First, the blockchain records and reveals all historical transactions with details to the owner, thereby enhancing the gemstone's traceability and visibility of all value creation steps. Moreover, the blockchain enables to store this information immutably and securely, providing a single source of truth. In addition, Gübelin Gem Lab offers to tag, i.e., emeralds with nanoparticles to create a unique identifier for each mine. By storing this identifier in the blockchain, each digital record can be verified by matching the record with the physical stone. Second, the blockchain application is designed to account for the heterogeneity of all actors in the gemstone industry and their context. For instance, rural mines might have limited access to wifi or phones with less image

\footnotetext{
${ }^{3}$ https://www.provenanceproof.com/
} 
resolution. Hence, the data can be uploaded at a later stage (e.g., by the trader). Thereby, the task to allow enhanced transparency within this context is supported by the technology. By design, blockchain allows the addition of new transaction data, e.g., the photography of a stone, and to trace back each entry. Therefore, manipulated information can always be traced back to its originator and thus penalizing its actions. Consequently, the technology is designed to increase the integrity of the data. For all owners of the stone, including temporary owners such as traders, the Provenance Proof Blockchain creates transparency, authenticity, and integrity as a value in an industry that has suffered from these regards. Both seller and buyer of a gemstone at each stage are now able to disclose relevant information on the origin and journey of the stone, leading to more trust on both transaction sides as a key value.

Network: Provenance Proof is a permissioned blockchain. Each actor in the colored gemstone industry that aims to read/write/verify has to undergo a know your customer (KYC) process before joining the initiative. After joining, each actor can choose to contribute to the consensus mechanism, enabling also smaller organizations to join without having to invest in additional resources. Actors that aim only at reading the information on a particular stone are able to do so without having to pass a KYC process. As a consequence, a network is established across the entire value chain from mine to end-customer. Especially downstream actors such as end-customers, retail brands, jeweler manufacturers gain a far better overview of the entire value chain up to their stage. Previously, the industry's information flow was limited to subsequent stages. The network of Provenance Proof allows information to flow across multiple stages, offering the opportunity to build new relationships with previous stages in the supply chain. The higher the number of actors in the industry, the more relations will be formed, increasing the value and opportunity for all entities.

\subsection{Case Study: TradeLens in shipping industry}

Industry: World trade is fueled by container ships that transports a large portion of world trade from the origin, e.g., in China, to destinations of consumers, e.g., in Europe. Goods stored in standard containers shipped internationally via the sea accounts for 80 of world trade. The transport via sea cost less than via air and land; however, the industry is challenged with more than $20 \%$ administrative cost and relatively poor performance even there is high competition among service providers.

Blockchain application: TradeLens is a blockchain technology-based platform for sharing shipping information targeted international shipping in containers. The majority of shipping lines within containerized shipping whom in total ships $66 \%$ of world traded transported in containers are committed to TradeLens. TradeLens was initiated by the market-leading shipping line Maersk (based on industry research) and piloted with IBM as the technology provider of the open-source Hyperledger Fabric (there are more than twenty competing technologies prototyped within the shipping industry). Later expanded to a consortium including three of its main competitors in the industry.

Technology fit: The fit between the task and the technology for the individual user is similar since the user use the same user interaction of their organizations legacy system but the value for both the user and the organization is increased by the interfaces to the blockchain, since that through API's provide real-time information directly from the source being other organisations in the supply chain, accordingly, e.g. the actual location and document approval status whereby logistic coordination can be improved accordingly the value provided when utilizing blockchain is significantly enhanced.

At the network level, the shipping involves more than 30 organizations that have digitized their information within their legacy systems. The result is several distributed information islands which are not interlinked. Blockchain technology is by design distributed where each node holds information about the events published by others. Therefore at the network level, the fit between the task and the technology can be characterized as mirroring the information islands spread globally across the organizations involved in international shipping and the distributed design globally on the internet across the nodes of blockchain technology. The blockchain technology builds on a distributed ledger for key events, e.g., export certificate document approved and container loaded on a specific container vessel, including more than 200 standardized events per container journey. Those events are published by more than 40 different organizations involved in the journey, from shippers ordering the transport, service providers loading the container, transporting, etc. and authorities approving (or rejecting) the movements. Having all those organizations publishing events for the whole journey from origin to destination creates a network effect resulting in a transparent supply chain where proactive actions can be taken to improve performance. The primary purpose and value that blockchain potentially brings are not so much tracking the supply chain of containerized 
shipping since that's already possible (through event information provided by shipping lines and forwarders for selected key events) to a large extend but rather the transparency opening insight into the administration of the containers (which prevents it from being moved on); secondly, the possibilities of automation of manual administrative tasks with the smart contract features of blockchain technology was the convincing argument for the investment of the CFO at Maersk, which would strategically impact the shipping line's competitive position and the industry performance as such.

Network: The ecosystem of maritime shipping can be described as supply chains for the physical transport and as networks particularly when including the administration. Within each specific of the supply chains, each organization is autonomous with a well-defined role, e.g., the shipping company transports the containers at sea, the terminal operator lifts the containers on and off vessels and trucks, and trucking companies transport the containers at land. Authorities govern, e.g., the containers when at ports. The flow, including information between the actors in the supply chain, is characterized as pier to pier, and each organization is reluctant to share information for various reasons. Accordingly, embeddedness as network density and the interconnection among the actors in the network is very fragmented and with separated organizations and no central actor. The blockchain is permissioned, wherefore every event is published by a trusted organization; accordingly, the proof of work is limited to the cryptographic part, which requires little energy compared to permissionless blockchains (used by, e.g., crypto-currencies). Further, the design of equal powered nodes in the blockchain respects and supports the autonomy of organizations involved in international shipping, ranging from shippers to competing service companies and to powerful authorities.

Initially, Maersk took the lead and, in a joint venture with the technology provider, offered the TradeLens solution to other organizations commercially. Maersk already had close collaboration and alliances with service providers (owner of some) and competing shipping lines and authorities. We conclude the structural embeddedness is high within the maritime shipping; however, the network effects creating business value did not appear since only a limited number of organizations joined TradeLens. After two years without additional major organizations joined TradeLens and their competitors initiating alternative blockchain solutions, Maersk gave up and invited their competitors on board, offering them both control and share in future business value, which resulted in the majority of leading shipping lines committing to
TradeLens. Accordingly, TradeLens has a commitment from container shipping lines transporting $2 / 3$ of world trade, which can generate significant both network effect and business value when digital interfaces are implemented. Remark that the interfaces between the individual organizations' legacy systems and TradeLens take time (years) to develop, wherefore the business value is yet to be proven.

Initially, the blockchain nodes are allocated to channels where each shipping line is the primary responsible. Accordingly, the consensus is handled firstly by the node owner but can be questioned and challenged by others. The consortium will when the blockchain solution is adapted (the primary task is implementing the API integration to legacy systems) control a significant share of world trade to the extent that it could become a de facto standard, causing a positive network effect with large coverage. Such a standard can improve the performance of the international containerized shipping via sea to the benefits of both shippers and the industry as such; additionally, the authorities can benefit significantly from the transparency into shipping information.

\section{Analysis and Discussion}

We divided the analysis and discussion into the three drivers task value, network value, and relationship value that were elaborated in the theoretical background.

\subsection{Task Value}

In both cases, blockchain technology is well suited to meet the requested task. In the Gemstone case, blockchain enables transparency, authenticity, and integrity in the industry that suffered from these regards. This creates particular value for sellers and buyers of a gemstone at each stage, especially for downstream parties close to ending customers. In the TradeLens case, blockchain enables sharing shipping information in real-time across the supply chain and promises transparency, allowing for better coordination and reduced cost. In its later phase, it is augmented towards smart contracts for automation of administration. Transparency provides direct value for shippers that may be indirectly absorbed by shipping lines and service providers. In the later phase, are also authorities included. The whole industry is supposed to benefit from resulting efficiency gains.

\subsection{Network value}

Both cases reveal network externalities that drive the dynamic development of the networks. Negative 
externalities refer to potential dependencies that appear in both cases, primarily in the early phase of the projects. In the TradeLens case, this is, e.g., dependent on IBM and Maersk in terms of the blockchain application. In the Gemstone case, the technological dependency on Everledger as a technology provider and the provider of nanoparticles (product tags) implies a lock-in in terms of technology and network dependency. Furthermore, a growing network with a larger number of actors limits the pace of refining the application, which is currently addressed in the Gemstone case by defining a board of governance. However, as the value of transparency depends on the scope and size of the network as well as the number of involved actors, there are apparent positive externalities. The gapless traceability of gemstones increases with the number of involved parties. Moreover, sourcing opportunities increased for downstream actors (e.g., jewelry manufacturers) due to additional relationships across multiple value stages.

Hence, there is additional value stemming from the network and the network effect itself that increases the value of the applications and thus creating dynamics for upscaling of the blockchain applications. In the TradeLens case that reached up to two-thirds of the relevant market share at the time of this writing and almost becomes a defacto standard, this appears even more significant than for the Gemstone case in an industry, which is more heterogeneous and permeable in its setup. But also, in the Gemstone case, the adoption increased from originally a Kernel with few bigger miners to now 320 actors adopted solution, spanning across all stages and different company sizes in the industry. However, both cases reveal restrictions that have been hindering the exploitation of the technology value. Both cases exhibit similar patterns of behavior for different groups of actors. In line with propositions of Gnyawali and Madhavan [8], we observe a central actor as initiators of the blockchain (Maersk and Gübelin Gem Lab). Though the technology value is apparent and network value is assumed, this action is responded by the other actors with hesitation towards collaboration up to even joining or creating competing blockchains. Structural embeddedness provides a relevant lens for explaining these restrictions, the resulting predictions of actor behavior and relationship value.

\subsection{Relationship value}

On a firm level, embeddedness is operationalized based on the centrality, and autonomy of the actors, and on network level based on equivalence and density. We use a relative scale of High, Medium and Low to compare the early and current stages for the cases and their industries. Table 1 summarizes the insights on the relationship value.

Centrality: refers to actors' different positions based on their roles in the network. In both cases, we see a confirmative pattern to the proposition of Gnyawali and Madhavan [8] that it is most likely a central actor that initiates action and that lower centrality of other actors restricts their ability to respond equally. TradeLens had in its first setup a central actor with the periphery of other actors. Proposing TradeLens as a service platform was putting the carrier Maersk as a central actor into a stronger competitive position than other actors in the same network echelon of the industry. The other carriers did not collaborate but tried with limited success to maintain their own central position with their own creation of alternative blockchains. In the new form, the blockchain becomes more of a polycentral construct. The initiator is less central and acting like the other carriers as polycentric multiplicators that bring their network to authorities and customers. This required a different organization of roles into a form of allied governance, including the sharing of the business value, i.e., profits from TradeLens. The Provenance Proof blockchain was also initiated by a central actor Gübelin Gem Labs. For the adopters, the participation in the network is increasing centrality for other actors compared to those that are not part of the blockchain and in comparison to their previous position.

The impact of actors' specific positions, expressed via centrality, is closely related to the structural autonomy of the actor. Structural autonomy refers to Burts [2] concept of structural holes. Structurally autonomous actors have structural holes between the actors they are connected to but are free of structural holes at their own end. Especially networks rich in structural holes opens opportunities for shifting control toward a focal actor that is more autonomous than the others [8]. We do not see this as a matter of the absolute degree of autonomy but more on autonomy gradients among actors - particularly those of similar types such as the carriers in the TradeLens Case or more prominent retail brands in the gemstone industry. In a focal actor-oriented approach, such as, for example, a control tower but also the original setup of TradeLens, designed as a commercial service, might increase structural autonomy for one actor but reduce it for collaborators and thus create or increase an autonomy gradient.

In the TradeLens case, carriers are connected to authorities and shippers but not connected to other carriers as these are competitors. Although lately several alliances were founded, the connection of these alliances is rare. Instead, an alliance is competing with an alliance. Overall, there remain structural 
holes horizontally between carriers, or at least between carriers of different alliances, and there is more freedom of structural holes in the more vertical view of the network. This means there is high autonomy among the carriers in the industry. Other levels, such as the forwarders, show a similar structure. This autonomy structure was not well represented by the first setup of TradeLens with Maersk as a central service provider but simultaneously also service user that is in competition to the other carriers.

Autonomy: Collaborating would imply for the other carriers the risk of sacrificing their structural autonomy. According to embeddedness theory, this asymmetry in autonomy weakening less autonomous actors in their ability to respond. They only can avoid this for the blockchain by not joining and competing with their own blockchains. By changing to a more polycentral structure, the structural holes between actors on the same level or between alliances remain similar for all of the actors, as before. Carriers continue to act autonomously but now act as multipliers to authorities and shippers instead of "sacrificing" links to those and thus their autonomy to one focal node.

In the same way, the gemstone industry exhibits structural holes between horizontal actors such as miners, cutters, or retail brands. However, in contrast to TradeLens, the initiator, Gübelin Gem Lab, had a high degree of autonomy from the start, having tiny structural holes in all directions of the value chain. While none of the competing gem labs joined the solution, actors on all other value stages joined the network, increasing their autonomy by gaining additional. The structural autonomy was key for the initiator, helping to increase the number of adopters by drawing on the direct ties to other actors on all value stages. For the joining actors, connecting via the blockchain also indirectly bridged structural holes due to a lack of business relationships.

Equivalence refers now to pairwise comparisons of actors and the similarity of their network patterns. Structural equivalent firms are viewed as having similar assets, information, and status flows. And as a result, behave similarly. To some extent, this links to mimicking mechanisms as known from institutional theory [5]. The Gemstone case is characterized by heterogeneous actors such as miners, retailers, etc. In this diverse ecosystem, we observe a pattern of behavior related to actor size, as in the beginning large miners almost exclusively use the blockchain. On the one hand, this would imply a rather equivalent network inside the blockchain and patterns of behavior. Large miners join, and the small firms stay outside. On the other hand, this indicates in regards to the whole industry a rather high lack of equivalence that restricts upscaling and adoption. We thus see in the beginning a low level of equivalence that divides large, international actors in one group and small, regional actors in the other group. With increased autonomy and shared centrality in the later phases, increased equivalence is found by allowing even smaller, regional actors to build up technology-based transparency. TradeLens initially shows different user groups with different fees. But even more interesting is the lacking equivalence as expressed by blockchain provider vs. blockchain user.

Based on the two cases, we propose that blockchains enable more equivalence under the premise that the connection is not based on the original business relationship but with a functional reference, creating transparency for the industry and when the actors are on an equal footing in terms of their function. Being a service provider and simultaneously service user created inequality in the TradeLens case. There is reduced inequality between producer or provider or appropriator of the information in its new form with open standards and profit-sharing. However, there is also a dark side of equivalence that leads to a dilemma. The more equal, the less likely it is, according to the theory, that one takes action. This might be a restriction towards upscaling and further development of the network and the application. Without inequality, in the beginning, there might be no initiative, and the more equalized the network is the slower it might develop.

Density is finally an overall network property related to the total network and refers to the interconnectedness among the actors in the network. The likelihood of action and response in a dense network differs from that in a less dense network. According to the theory, f.i. does higher density diminish centrality effects and does, in general, dampen competitive effects. Density is thus a kind of context-related moderator of the other influencing factor for behavior.

TradeLens case shows heterogeneous actor types with limited horizontal links within types due to competition and links in vertical channels for transactions. The gemstone industry is even more unconnected due to the higher number of different actors and their size. Both blockchains are permission-based, creating a higher density within the network and a density gradient between inside and outside the network. Being part of the blockchain provides actors with information access they would not have without the blockchain. The more actors are in the blockchain, and the higher is the effect. Here the embeddedness views push back on the network effects. In the Gemstone case, in particular, downstream actors get an indirect connection to upstream actors they are in fact not connected with. Similar indirect connections 


\begin{tabular}{|c|c|c|c|c|}
\hline & \multicolumn{2}{|c|}{ Colored Gemstone Case } & \multicolumn{2}{|c|}{ TradeLens Case } \\
\hline & Early stage & Current stage & Early stage & Current stage \\
\hline \multicolumn{5}{|c|}{ Centrality - firm level } \\
\hline $\begin{array}{l}\text { Industry } \\
\text { network }\end{array}$ & \multicolumn{2}{|c|}{ Medium - Few players with high centrality } & \multicolumn{2}{|c|}{$\begin{array}{l}\text { High - But oligopolistic with } 3 \text { market leading companies } \\
\text { collaborating in alliances }\end{array}$} \\
\hline $\begin{array}{l}\text { Blockchain } \\
\text { network }\end{array}$ & $\begin{array}{l}\text { High - For central } \\
\text { initiator } \\
\text { Low - For peripheral } \\
\text { adopters }\end{array}$ & $\begin{array}{l}\text { High - Increased } \\
\text { centrality for all } \\
\text { actors by increased } \\
\text { information flow }\end{array}$ & $\begin{array}{l}\text { High - For central initiator } \\
\text { Low - For minor peripheral } \\
\text { adopters }\end{array}$ & $\begin{array}{l}\text { High - With market } \\
\text { leading companies, sharing } \\
\text { governance and business } \\
\text { value }\end{array}$ \\
\hline \multicolumn{5}{|c|}{ Autonomy - firm level } \\
\hline $\begin{array}{l}\text { Industry } \\
\text { network }\end{array}$ & \multicolumn{2}{|c|}{$\begin{array}{l}\text { Medium - Many actors with structural } \\
\text { holes, few without }\end{array}$} & $\begin{array}{l}\text { High - Efficient physical } \\
\text { supply chain }\end{array}$ & $\begin{array}{l}\text { High - Increased for all by } \\
\text { additional relationships }\end{array}$ \\
\hline $\begin{array}{l}\text { Blockchain } \\
\text { network }\end{array}$ & $\begin{array}{l}\text { High - For initiator } \\
\text { and a few miners } \\
\text { Low - For other } \\
\text { adopters }\end{array}$ & $\begin{array}{l}\text { High - Increased } \\
\text { for all by additional } \\
\text { relationships }\end{array}$ & $\begin{array}{l}\text { High - For the sea part of the } \\
\text { shipments and, the initiating } \\
\text { shipping line } \\
\text { Low - for the other adopters }\end{array}$ & $\begin{array}{l}\text { High - TradeLens } \\
\text { gain transparency acrosts } \\
\text { extended supply chain } \\
\text { (beyond sea part). }\end{array}$ \\
\hline \multicolumn{5}{|c|}{ Equivalence - in pairwise comparison of types of actors } \\
\hline $\begin{array}{l}\text { Industry } \\
\text { network }\end{array}$ & \multicolumn{2}{|c|}{$\begin{array}{l}\text { Low - Large international actors, small } \\
\text { regional actors in the other }\end{array}$} & \multicolumn{2}{|c|}{$\begin{array}{l}\text { Low - Between private companies and national authorities } \\
\text { High For competing private organisations along the supply } \\
\text { chain (Carriers, Forwarders, and other service providers) }\end{array}$} \\
\hline $\begin{array}{l}\text { Blockchain } \\
\text { network }\end{array}$ & $\begin{array}{l}\text { Low } \quad-\quad \text { Large } \\
\text { international initiator } \\
\text { and large mines }\end{array}$ & $\begin{array}{l}\text { Medium - Increased } \\
\text { "functional" } \\
\text { equivalence, smaller } \\
\text { actors with same } \\
\text { functions }\end{array}$ & $\begin{array}{l}\text { Low - Initiator different to } \\
\text { other users and technology } \\
\text { provider having monopolistic } \\
\text { position }\end{array}$ & $\begin{array}{l}\text { High - Due to open } \\
\text { standards, a reference } \\
\text { board; and to sharing } \\
\text { business value (between the } \\
\text { extended initiator group of } \\
\text { three market leaders). }\end{array}$ \\
\hline \multicolumn{5}{|c|}{ Density - on network level } \\
\hline $\begin{array}{l}\text { Industry } \\
\text { network }\end{array}$ & $\begin{array}{l}\text { Low - A few actors } \\
\text { have numerous ties }\end{array}$ & $\begin{array}{l}\text { High - Increased } \\
\text { density by indirect } \\
\text { ties for all adopting }\end{array}$ & $\begin{array}{l}\text { Low - Actors connected } \\
\text { peer-to-peer vertically but } \\
\text { less horizontally }\end{array}$ & $\begin{array}{lr}\text { High - Increased } & \text { indirect } \\
\text { interconnection } & \text { via } \\
\text { multipliers } & \end{array}$ \\
\hline $\begin{array}{l}\text { Blockchain } \\
\text { network }\end{array}$ & $\begin{array}{l}\text { Medium - Permission } \\
\text { based, high density } \\
\text { only for initiator }\end{array}$ & $\begin{array}{l}\text { High - Increased } \\
\text { density by increased } \\
\text { indirect ties for all } \\
\text { actors }\end{array}$ & $\begin{array}{l}\text { Medium - Permission } \\
\text { based, high density only } \\
\text { among within a blockchain } \\
\text { competing blockchain }\end{array}$ & $\begin{array}{l}\text { High - Increased density and } \\
\text { network effects by indirect } \\
\text { ties across supply chains } \\
\text { network. }\end{array}$ \\
\hline
\end{tabular}

Table 1. Relationship Value related properties: Embeddedness

happen in the TradeLens case.

Our proposition is that the blockchain does not necessarily increase the interconnectedness of the industry, but it creates indirect interconnection for actors that are a member of the blockchain. This creates higher density within the blockchain as compared to outside the blockchain. However, this interconnection is reduced to functional aspects of transparency but not necessarily linked to the commercial connections among actors. Competitive elements are thus not necessarily affected.

\section{Conclusion}

Based on our investigation and analysis of the two cases, we explored that - beyond technology fit - the network fit is key to the success of blockchain technology. While the fit between task and technology represents a necessary condition for blockchain adoption and use, the network value and relationship value ultimately drive blockchain networks' success. From our analysis of the supply chain cases in two different industries, we reveal a good task and technology fit in both cases. However, they differ in terms of the network value and the relationship value over time. Blockchain technology is well suited to integrating the supply chain from end to end, creating transparency across the organization's chain with their distributed information silos. The solution meets the requested task to create transparency in real-time. The Gemstone case is very successful in covering the complete supply chain, including all stages of the industry with a large number of actors in the blockchain solutions. Similarly, for the TradeLens case, the extended supply chain was initiated by Maersk being the market leader with $15 \%$ market share and a few minor partners; in the early years, TradeLens struggled 
lacking commitment from other than the initiator's close partners. As the owner of TradeLens in 2019, Maersk gave in governance to the competitors, e.g., the other major shipping lines who then committed to join TradeLens whereby TradeLens potentially facilitate $2 / 3$ of the world transports in containers. Our analysis finds that beyond the task technology fit, the centrality and autonomy of the initiator is a good starting position to launch the blockchain at an early phase. However, the cases reveal that by remaining the only central and autonomous player, the initiator cannot increase adoption success in the long run. Hence, in order to increase the adoption success and extend the network along the supply chain, the initiator has to increase the autonomy, equivalence, and density of the organizations in the network and potential adopters to enhance network value and relationship value for these actors as well. Thus, only focusing on improving the fit between technological features and the resulting task technology value will not be sufficient. Our investigation of the two cases reveals that fit means complementing the task technology fit with network fit. Accordingly, initiators, technology providers, and early-stage adopters have to look for ways to increase the network value and relationship value. Future research can validate with increasing number of cases possibly across additional types of supply chains and industries e.g for pharmaceuticals or industries with high intellectual capital expanding on the task technology fit model in networks, including the concept of structural embeddedness.

\section{References}

[1] G. Blossey, J. Eisenhardt, and G. Hahn, "Blockchain technology in supply chain management: an application perspective," in Proceedings of the 52nd Hawaii international conference on system sciences, 2019.

[2] R. S. Burt, Structural holes: The social structure of competition. Harvard university press, 2009.

[3] R. Cole, M. Stevenson, and J. Aitken, "Blockchain technology: implications for operations and supply chain management," Supply Chain Management: An International Journal, 2019.

[4] F. D. Davis, "Perceived usefulness, perceived ease of use, and user acceptance of information technology," MIS quarterly, 1989, 319-340.

[5] P. J. DiMaggio and W. W. Powell, "The iron cage revisited: Institutional isomorphism and collective rationality in organizational fields," American sociological review, 1983, 147-160.

[6] M. C. Dong, Y. Fang, and D. W. Straub, "The impact of institutional distance on the joint performance of collaborating firms: The role of adaptive interorganizational systems," Information Systems Research, 28, 2017, no. 2, 309-331.

[7] S. Farshidi, S. Jansen, S. España, and J. Verkleij, "Decision support for blockchain platform selection:
Three industry case studies," IEEE Transactions on Engineering Management, 67, 2020, no. 4, 1109-1128.

[8] D. R. Gnyawali and R. Madhavan, "Cooperative networks and competitive dynamics: A structural embeddedness perspective," Academy of Management review, 26, 2001, no. 3, 431-445.

[9] D. L. Goodhue and R. L. Thompson, "Task-technology fit and individual performance," MIS quarterly, 1995.

[10] L.-V. Herm and C. Janiesch, "Towards an implementation of blockchain-based collaboration platforms in supply chain networks: A requirements analysis," in Proceedings of the 54th Hawaii International Conference on System Sciences, 2021.

[11] M. L. Katz and C. Shapiro, "Technology adoption in the presence of network externalities," Journal of political economy, 94, 1986, no. 4, 822-841.

[12] T. K. Mackey and G. Nayyar, "A review of existing and emerging digital technologies to combat the global trade in fake medicines," Expert opinion on drug safety, 16, 2017, no. 5, 587-602.

[13] S. Mazumdar, T. Jensen, R. R. Mukkamala, R. Kauffman, and J. Damsgaard, "Do blockchain and iot architecture create informedness to support provenance tracking in the product lifecycle?" in Proceedings of the 54th Hawaii International Conference on System Sciences. Hawaii International Conference on System Sciences (HICSS), 2021, 1497-1506.

[14] B. Müßigmann, H. von der Gracht, and E. Hartmann, "Blockchain technology in logistics and supply chain management-a bibliometric literature review from 2016 to january 2020," IEEE Transactions on Engineering Management, 67, 2020, no. 4, 988-1007.

[15] A. Ravald and C. Grönroos, "The value concept and relationship marketing," European journal of marketing, 1996.

[16] S. Saberi, M. Kouhizadeh, J. Sarkis, and L. Shen, "Blockchain technology and its relationships to sustainable supply chain management," International Journal of Production Research, 57, 2019, no. 7.

[17] S. Seebacher and M. Maleshkova, "A model-driven approach for the description of blockchain business networks," in Proceedings of the 51st Hawaii International Conference on System Sciences, 2018.

[18] P. Shortell and E. Irwin, "Governing the gemstone sector: Lessons from global experience," Natural Resource Governance Institute. UK Department of International Development and Australian Department of Foreign Affairs and Trade, 2017.

[19] H. S. Sternberg, E. Hofmann, and D. Roeck, "The struggle is real: Insights from a supply chain blockchain case," Journal of Business Logistics, 42, 2021, no. 1.

[20] C. Straubert, E. Sucky, and J. Mattke, "Blockchain technology for tracking and tracing in supply chains: A critical viewpoint," in Proceedings of the 54th Hawaii International Conference on System Sciences, 2021.

[21] A. H. Van de Ven and M. S. Poole, "Alternative approaches for studying organizational change," Organization studies, 26, 2005, no. 9, 1377-1404.

[22] V. Venkatesh, M. G. Morris, G. B. Davis, and F. D. Davis, "User acceptance of information technology: Toward a unified view," MIS quarterly, 2003, 425-478.

[23] I. Zigurs and B. K. Buckland, "A theory of task/technology fit and group support systems effectiveness," MIS quarterly, 1998, 313-334. 